\title{
Diffusion tensor imaging investigations in Alzheimer's disease: the resurgence of white matter compromise in the cortical dysfunction of the aging brain
}

\author{
David A Medina' \\ Moises Gaviria² \\ 'Department of Psychiatry, \\ Case Western Reserve University, \\ Cleveland, OH, USA; ${ }^{2}$ University \\ of Illinois at Chicago, IL, USA
}

Correspondence: David A Medina Department of Psychiatry, University Hospitals Case Medical Center, I I 100 Euclid Avenue, Cleveland, OH 44I06, USA Email davimedin@hotmail.com

\begin{abstract}
Diffusion tensor imaging (DTI) is a sophisticated MRI-based neuroimaging technique that enables in vivo quantification of differences in molecular diffusion at the cellular level. Owing to the highly directional architecture of white matter (WM), DTI is providing important clues of the structure and geometric organization of this neural compartment. Since DTI can detect changes even in the case of radiologically "normal" appearing WM, researchers are using the technique for the study of WM integrity at the initial stages of the most common neurodegenerative disorders. Along with a well characterized cortical pathology (neuritic plaques and intracellular neurofibrillary tangles), WM changes have been also demonstrated in Alzheimer's disease (AD). However, these changes had been for years found nonliable in the onset and progress of $\mathrm{AD}$, basically due to lack of incriminatory evidence. The use of novel tools such as DTI has enabled the anatomical distribution of WM microstructural damage in the prodromal stages of $\mathrm{AD}$ to be gauged and determined, granting a long-delayed protagonic role to WM in the natural history of this highly prevalent neurodegenerative condition.
\end{abstract}

Keywords: diffusion, DTI, white matter, Alzheimer's disease, aging, anisotropy

The study of molecular diffusion by means of magnetic resonance imaging (MRI) has had a great impact in modern neuroscience. An MRI-based technique called diffusion weighted imaging (DWI) is perhaps one of the best examples of translational research from the basic sciences to the clinical arena - exploiting changes in cellular water diffusion properties and providing key information on the actual physiological state of brain tissue beyond the purely "anatomical" data. DWI enables a robust visualization of abnormalities within minutes of onset of cerebral ischemia (Moseley et al 1990; Hossmann and Hoehn-Berlage 1995; Lutsep et al 1997; Yoneda et al 1999; Lansberg et al 2000), as well as white matter (WM) microstructural changes in several neurological conditions (Higano et al 2001), including the dementia syndrome caused by Alzheimer's disease (Hanyu et al 1998, 1999; Sandson et al 1999; Bozzao et al 2001; Kantarci et al 2001).

When physical barriers are absent, diffusion is isotropic, ie, equal in every direction. However, the intrinsic peculiarities of biological microenvironments work as constrictors of free water diffusion, obligating molecules to flow in a directional or anisotropic fashion. Despite its well deserved position as the standard imaging aid for the diagnosis and management of acute stroke (Neumann-Haefelin et al 2000; Lansberg et al 2001), the information obtained from conventional DWI is limited to one dimension, based only on intensity values. To overcome this limitation, investigators 
engineered a sophisticated MRI modality called diffusion tensor imaging (DTI; Le Bihan et al 2001). DTI quantifies molecular motion at the voxel level by means of a tensor, a mathematical concept that allows for complete tridimensional description of both the direction and the average magnitude of water diffusion (Basser et al 1994a, b; Basser 1995).

The structure of WM consists mainly of highly organized, directionally insulated tracts formed by myelin-covered axons. Hence, diffusion anisotropy is more likely to be higher in WM than in other tissues, eg, gray matter (GM). DTI measures of WM anisotropy such as fractional anisotropy (FA; Basser and Pierpaoli 1996) are currently regarded as markers of the integrity of neural axons (Beaulieu 2002), positioning the technique as a reliable indicator of defects in geometric organization and fiber architecture in the context of disease. DTI has the advantage of examining the microstructural integrity of even radiologically "normal" appearing WM, enabling assessment of the status of WM at the initial stages of the most common neurodegenerative disorders.

\section{Use of DTI to address the "dementia problem"}

Alzheimer's disease (AD) is pathologically defined by the presence of neuritic plaques, intracellular neurofibrillary tangles, and a greater loss of cortical neurons than in sameage, non-demented persons. Although they are most common in vascular dementia, independent white matter changes (WMC) have also been found in AD to a greater extent than in age-matched controls (Brun and Englund 1986; Englund 1998; Kalaria 2000, 2002; Bronge et al 2002). However, the role of WMC in the pathogenesis, disease progression, and cognitive features of $\mathrm{AD}$ remained unsolved.

Mild cognitive impairment (MCI) is usually defined as a slight impairment in cognitive function - memory, in the case of amnestic MCI - but lacking enough criteria to be considered full dementia. MCI has become a common diagnosis in later life and is associated with a high risk of developing $\mathrm{AD}$, with yearly conversion rates estimated at $10 \%-15 \%$ (Petersen et al 2001). In vivo anatomical markers determined by quantitative MRI methods are being successfully used to differentiate patients with MCI and AD, respectively, from normal controls, and highly predictive indices of conversion (from MCI to AD) have been generated (deToledo-Morrell et al 2000, 2004; Killiany et al 2000, 2002; Dickerson et al 2001).

Although mesial temporal cortical structures involved in memory function, such as the hippocampus and entorhinal cortex, have been traditionally under the scope of AD research
(Jack et al 1992, 1997; Convit et al 1997; deToledo-Morrell et al 1997, 2000, 2004; Bobinski et al 1999; Killiany et al 2000, 2002; Xu et al 2000; Callen et al 2001; Dickerson et al 2001; Du et al 2001), DTI now is guiding scientists in their observations of the WM microstructural changes associated with aging and aging-related cognitive decline in the living brain. In AD in particular, the study of anisotropy changes has been focused on the prodromal and initial stages of the disease.

The localization of DTI-derived WM changes has been accomplished mostly by three methods: a) Selecting a region of interest (ROI), usually prescribed manually and from which anisotropy values are measured for statistical comparisons. b) Selecting a tract of interest from which the main eigenvector of diffusion, parallel to the major axis of the fiber bundle, is calculated. This method is called tractography and uses color codes and predetermined anatomical seeds (Conturo et al 1999; Pajevic and Pierpaoli 1999), to virtually dissect a complete WM tract from its origin to its termination in the brain (Virta et al 1999; Poupon et al 2000). c) More recently, fully automated whole-brain algorithms have been adopted to register individual anisotropy maps into standard space, and compute their changes by voxelbased statistics.

Using the ROI approach, several studies have looked at the WM of multiple brain lobes and some functionally relevant structures such as the superior longitudinal fasciculus, the cingulum bundle, and the corpus callosum. In summary, the majority of regional changes found in DTI indices of WM integrity are located posteriorly in the brains of MCI and AD subjects (Rose et al 2000; Bozzali et al 2002; Takahashi et al 2002; Yoshiura 2002; Fellgiebel et al 2004, 2007; Head et al 2004; Duan et al 2006; Naggara et al 2006; Huang and Auchus 2007; Huang et al 2007; Sydykova et al 2007). In contrast, effects of normal aging were found to be of greater magnitude in the WM of the frontal lobe and the anterior corpus callosum than in the WM of temporal, parietal, occipital, and posterior callosum regions (Head et al 2004).

Medina et al (2006) published an in vivo examination of normal-appearing WM microstructural integrity in individuals with MCI and mild AD using the whole brain approach for the first time, avoiding the biases of the a priori selection of ROIs. In this study, significant regional decreases in FA were found in the WM of the two patient groups, as opposed to age-matched healthy controls, in voxel-by-voxel comparisons. Interestingly, the anatomical pattern of FA changes was similar for both patient groups with greater posterior than anterior involvement. In a complementary experiment 
(Medina et al 2005), the same methodology was used to detect significant voxel-wise FA reductions in a group of old normal controls (ONC) versus young normal controls (YNC). When these results were compared with the findings of the first experiment, the anterior/posterior distribution of voxel-wise anisotropy reductions was significantly different between the ONC vs YNC comparisons and the MCI and AD vs ONC comparisons, with a greater percentage of posterior WM integrity loss in the cognitively affected patients. These results further contribute to the body of evidence in favor of a more pronounced posterior WM damage in cognitively impaired elderly individuals.

\section{What is DTI telling us?}

The DTI-detected regional WM alterations in AD seem to parallel the pattern of changes followed by the GM in the disease process, that is, cortical abnormalities are greater in posterior brain regions relative to anterior regions at the early stages of AD (Arnold et al 1991; Braak and Braak 1995). As the disease advances, the neurofibrillary pathology progresses from limbic to frontal structures, into high-order association cortices, and finally affects primary sensorimotor areas, which correlates with the clinical manifestations of AD. From post-mortem specimens to different imaging modalities, eg, MRI, PET, and SPECT, this typical sequence has been confirmed at different points of the disease (for a review, see Thompson et al 2007).

Fibers located deep in the posterior WM, such as the superior longitudinal fasciculus and the posterior cingulum bundle, are being consistently found to be affected in patients with $\mathrm{AD}$ and MCI, not only in ROI studies, but also in DTI-based tractography (Fellgiebel et al 2005; Xie et al 2005) and whole-brain DTI studies (Medina et al 2006; Rose et al 2006; Zhang et al 2007). A strong body of evidence has supported the early involvement of the posterior cingulate (PC) in the progression of AD. Histologic, volumetric (Callen et al 2001), and metabolic (Reiman et al 1996, 2001; Minoshima et al 1997; Valla et al 2001) data have shown that the $\mathrm{PC}$ is not only involved early in $\mathrm{AD}$, but it may also undergo changes even in asymptomatic genetically predisposed individuals (Reiman et al 2001). A plausible explanation for these phenomena has been proposed by Bartzokis (Bartzokis 2004; Bartzokis et al 2003, 2004). According to this model, as the brain development takes place, later myelinated regions (cortical association areas) have fewer oligodendrocytes supporting greater numbers of axons compared with earlier myelinated regions (primary sensorimotor regions). Oligodendrocytes in the cortical association areas have higher metabolic demands in order to maintain the widely distributed axons, making them more susceptible to pathological processes such as oxidative stress and accumulation of ferritin iron. These and other metabolic insults may lead to decreased WM integrity in association cortical regions. In general, the DTI findings of decreased WM integrity in later-myelinated regions at the onset of $\mathrm{AD}$ support this "reversed de-myelination" construct.

Cerebrovascular disease and WM dysfunction are inherently associated with vascular risk factors such as diabetes, myocardial infarction or hypertension. In addition to ischemic events, common in the elderly population, those factors seem to be also related to an increased risk of AD (Kalaria 2000, 2002; Urresta et al 2003). Thus, vascular pathology may be an additive element to the deleterious effects of aging in more vulnerable, long cortico-cortical WM tracts.

DTI has been successfully applied to the study of diseases causing axonal damage (Ulug et al 1999), identifying reductions in anisotropy not only in macroscopic WM lesions but also at the microscopic level (Stieltjes et al 2001). The DTI renditions of changes in cerebral diffusion occurring in the WM of stroke patients have made possible the discrimination in vivo between primary (direct) and secondary (Wallerian) degeneration (Makris et al 1997; Mukherjee et al 2000; Werring et al 2000; Pierpaoli et al 2001). In the case of direct damage to the WM, as liquefactive necrosis takes place and the content of isotropic free water, ie cerebrospinal fluid, increases in the axon, severe reductions in FA are accompanied by an increase in mean diffusivity - otherwise known as trace $D$ - as a result of a global increase in diffusivity in all directions. On the other hand, although Wallerian degeneration is also characterized by reduced FA and, albeit lesser, increases in mean diffusivity, there are differential changes in directional diffusivities: axial diffusivity (DA: diffusion parallel to the main axis of the tract) is reduced while radial diffusivity (DR: diffusion perpendicular to the main axis of the tract) remains high.

As mentioned in the previous section, there is plenty of pathoanatomical evidence of $\mathrm{WMC}$ in $\mathrm{AD}$, thought to be the result of a constellation of pathologies such as WM rarefaction and axonal gliosis, probably related to ischemia, and an anterograde Wallerian degeneration that may follow the stereotypic pattern of AD cortical atrophy. Consequently, DTI directional diffusivities have been used to elucidate the extent of Wallerian degeneration in the normal-appearing WM of MCI and mild AD patients (Huang and Auchus 200; Huang et al 2007). The most consistent findings of a pilot study using this approach (Huang et al 2007) were seen 
in the temporal lobes: both MCI and AD groups showed significant reductions in FA, but the MCI group showed only a decrease in DA - probably reflecting an incipient axonal damage in a vulnerable target area - while the AD group showed decreases in DA and increases in DR, suggesting a more advance state of coexistent axonal destruction and demyelination that is coupled with the characteristic pattern of AD cortical loss.

DTI investigators are also looking for correlations between their regional estimations of WM integrity and cognitive function. Relationships have been sought between scores of a widely used diagnostic instrument in dementia studies, the Mini Mental State Examination (MMSE: Folstein et al 1975), and WM mean diffusivity and anisotropy values, with findings of decreased cognitive status associated with decreased WM integrity in cerebral posterior regions of AD subjects (Rose et al 2000; Bozzali et al 2002; Yoshiura et al 2002; Duan et al 2006). Further research including amnestic MCI populations and utilizing appropriate neuropsychological tests of declarative memory, has demonstrated significant correlations between declining performance in these tasks and decreases in posterior WM anisotropy, particularly in the posterior cingulum bundles (Fellgiebel et al 2005, 2007; Rose et al 2006). Moreover, Huang et al (2007, see above) were able to delineate a neuroanatomical pattern of functional alterations in their cognitively impaired elderly subjects in such a way that changes in WM diffusion indices of the temporal lobes correlated with scores of episodic memory, WM diffusion changes in the frontal lobes correlated with scores of executive function, and WM diffusion changes in parietal lobes correlated with scores of visuospatial skills. This pattern might be indicative of the vulnerability of cortical cognitive systems to the deterioration of the WM pathways embedded beneath the disease-targeted gyri, and postulates disruptions in transcortical connectivity as an early contributor to the pathophysiology of dementia.

\section{What's coming next?}

In pursuit of a deeper understanding of WMC in AD, investigators are narrowing their scope into brain areas that are difficult to access by conventional neuroimaging tools. The perforant path (PP), a parahippocampal WM bundle that originates from cells in the entorhinal cortex and provides the hippocampus with its major cortical input, has been elusive to the DTI community due to the technical constrains of the ROI approach, particularly in terms of partial volume effects. In an effort to surmount these difficulties, high resolution DTI protocols are being used in combination with high resolution anatomical MRI scans to obtain in vivo evidence of reductions in WM volume and FA in parahippocampal regions of patients with AD (Wang et al 2006). A similar bimodal ROI approach had comparable findings in a DTI study of the PP that included AD and MCI patients (Kalus et al 2006), and through the benefit of an optimized voxel-based method, Rose and colleagues (2006) were able to replicate parahippocampal WM changes when they examined a group of MCI patients. Taken together, these results suggest that a disruption in the flow of information to the hippocampus may exist since the beginnings of $\mathrm{AD}$, giving WM dysfunction a refreshed protagonic role in the study of age-associated cognitive impairment.

Promising applications such as DTI-based tractography (DTT) or voxel-wise analyses are not free of limitations either. Apart from partial voluming effects, ubiquitous artifacts arise when a tract of interest is reconstructed through DTT, due to a problematic reproduction of the natural angulations of WM fibers. A recent advance in this topic is a provocative proposal by Yasmin et al (2008), in which a minimally operator-dependent optimized DTT technique, the "erosion" method, was used to detect neatly decreases in FA in the uncinate fasciculum of AD subjects. Problems in voxelwise studies are related to the coregistration of individual DTI maps to standard templates and their segmentation via probability masks. Defining the proper criteria for smoothing and thresholding of the images for statistical purposes are additional issues. Laudable initiatives towards a careful alignment of DTI images and the use of sample-specific averages instead of exogenous templates have replicated the previously reported WM anisotropy changes occurring in posterior WM regions of $\mathrm{AD}$ subjects (Rose et al 2008).

A new technical development called tract-based spatial statistics (TBSS: Smith et al 2006), which aimed to combine the strengths and to compensate for the weaknesses among the aforementioned methods, is being included in some of the commonly used open-source software tools for post-processing of DTI data. Instead of calculating the FA of a specific tract of interest, TBSS creates a "mean FA skeleton" from the very sample under investigation and later projects individual FA maps to this unique "skeleton-space", avoiding alignment and smoothing issues and permitting voxel-wise statistical comparisons among groups. Although yet to be seen in AD, this kind of solution will surely help to provide more precise information about the WM compromise in the malfunction of the aging brain.

Current multi-center collaborative initiatives such a the National Alliance for Medical Imaging Computer 
(www.na-mic.org) or the Biomedical Informatic Research Network (www.nbirn.net) are trying to develop automated image calibration and analysis tools to facilitate the gathering of larger amounts of DTI data (among other imaging modalities), and to make that information available to the research community in public databases. These projects are seeking to enhance the applicability of the technique, ie, to develop biomarkers suitable for the monitoring of therapeutic efficacy in clinical trials, or to identify patients at risk and susceptible to benefit from early pharmacological interventions.

\section{References}

Arnold SE, Hyman BT, Flory J, et al. 1991. The topographical and neuroanatomical distribution of neurofibrillary tangles and neuritic plaques in the cerebral cortex of patients with Alzheimer's disease. Cerebral Cortex, 1:103-16.

Bartzokis G. 2004. Age-related myelin breakdown: a developmental model of cognitive decline and Alzheimer's disease. Neurobiol Aging, 25:5-18.

Bartzokis G, Cummings JL, Sultzer D, et al. 2003. White matter structural integrity in healthy aging adults and patients with Alzheimer's disease. Arch Neurol, 60:393-8.

Bartzokis G, Sultzer D, Lu PH, et al. 2004. Heterogenous age related breakdown of white matter structural integrity: implications for cortical "disconnection" in aging and Alzheimer's disease. Neurobiol Aging, 25:843-51.

Basser PJ. 1995. Inferring microstructural features and the physiological state of tissues from diffusion-weighted images. NMR Biomed, 8:333-44.

Basser PJ, Mattiello J, LeBihan D. 1994a. MR diffusion tensor spectroscopy and imaging. Biophys $J, 66: 259-67$.

Basser PJ, Matiello J, Le Bihan D. 1994b. Estimation of the effective self-diffusion tensor from the NMR spin echo. J Magn Reson B, 103 247-54.

Basser PJ, Pierpaoli C. 1996. Microstructural and physiological features of tissues elucidated by quantitative-diffusion-tensor MRI. $J$ Magn Reson B, 111:209-19.

Beaulieu C. 2002. The basis of anisotropic water diffusion in the nervous system - a technical review. NMR Biomed, 15:435-55.

Bobinski M, de Leon MJ, Convit A, et al. 1999. MRI of entorhinal cortex in mild Alzheimer's disease. Lancet, 353:38-40.

Bozzali M, Falini A, Franceschi M, et al. 2002. White matter damage in Alzheimer's disease assessed in vivo using diffusion tensor magnetic tensor imaging. J Neurol Neurosurg Psychiatry, 72:742-6.

Bozzao A, Floris R, Baviera ME, et al. 2001. Diffusion and perfusion MR imaging in cases of Alzheimer's disease: correlations with cortical atrophy and lesion load. AJNR Am J Neuroradiol, 22:1030-6.

Braak H, Braak E. 1995. Staging of Alzheimer's disease-related neurofibrillary changes. Neurobiol Aging, 16:271-8.

Bronge L, Bogdanovic N, Wahlund LO. 2002. Postmortem MRI and histopathology of white matter changes in Alzheimer brains. Dement Geriatr Cogn Disord, 13:205-12.

Brun A, Englund E. 1986. A white matter disorder in dementia of the Alzheimer type: a pathoanatomical study. Ann Neurol, 19:253-62.

Callen DJA, Black SE, Gao F, et al. .2001. Beyond the hippocampus: MRI volumetry confirms widespread limbic atrophy in AD. Neurology, 57:1669-74.

Conturo TE, Lori NF, Cull TS, et al. 1999. Tracking neuronal fiber pathways in the living human brain. Proc Natl Acad Sci USA, 96:10422-7.

Convit A, de Leon MJ, Tarshish C, et al. 1997. Specific hippocampal volume reductions in individuals at risk for Alzheimer's disease. Neurobiol Aging, 18:131-18.
deToledo-Morrell L, Goncharova I, Dickerson B, et al. 2000. From healthy aging to early Alzheimer's disease. In vivo detection of entorhinal cortex atrophy. Ann NY Acad Sci, 911:240-53.

deToledo-Morrell L, Stoub TR, Bulgakova M, et al. 2004. MRI-derived entorhinal volume is a good predictor of conversion from MCI to AD. Neurobiol Aging, 25:1197-203.

deToledo-Morrell L, Sullivan MP, Morrell F, et al. 1997. Alzheimer's disease: In vivo detection of differential vulnerability of brain regions. Neurobiol Aging, 18:463-8.

Dickerson BC, Goncharova I, Sullivan MP, et al. 2001. MRI-derived entorhinal and hippocampal atrophy in incipient and very mild Alzheimer's disease. Neurobiol Aging, 22:747-54.

Du AT, Schuff N, Amend D, et al. 2001. Magnetic resonance imaging of the entorhinal cortex and hippocampus in mild cognitive impairment and Alzheimer's disease. J Neurol Neurosurg Psychiatry, 71:441-7.

Duan JH, Wang HQ, Xu J, et al. 2006. White matter damage of patients with Alzheimer's disease correlated with the decreased cognitive function. Surg Radiol Anat, 28:150-6.

Englund E. 1998. Neuropathology of white matter changes in Alzheimer's disease and vascular dementia. Dement Geriatr Cogn Disord, 9 (Suppl 1):6-12.

Fellgiebel A, Müller MJ, Wille P, et al. 2005. Color-coded diffusion-tensorimaging of posterior cingulate fiber tracts in mild cognitive impairment. Neurobiol Aging, 26:1193-8.

Fellgiebel A, Schermuly I, Gerhard A, et al. 2007. Functional relevant loss of long association fibre tracts integrity in early Alzheimer's disease. Neuropsychologia. [Dec 23 Epub ahead of print].

Fellgiebel A, Wille P, Müller MJ, et al. 2004. Ultrastructural hippocampal and white matter alterations in Mild Cognitive Impairment: a diffusion tensor imaging study. Dement Geriatr Cogn Disord, 18:101-8.

Folstein MF, Folstein SE, McHugh PR. 1975. Mini-Mental State. A Practical method for grading the mental status of patients for the clinician. $J$ Psychiatric Res, 12:189-92.

Hanyu H, Asano T, Sakurai H, et al. 1999. Diffusion-weighted and magnetization transfer imaging of the corpus callosum in Alzheimer's disease. $J$ Neurol Sci, 167:37-44.

Hanyu H, Sakurai H, Iwamoto T, et al. 1998 Diffusion-weigthed MR imaging of the hippocampus and temporal white matter in Alzheimer's Disease. J Neurol Sci, 156:195-200.

Head D, Buckner RL, Shimony JS, et al. 2004. Differential vulnerability of anterior white matter in nondemented aging with minimal acceleration in dementia of the Alzheimer type: Evidence from diffusion tensor imaging. Cereb Cortex, 14:410-23.

Higano S, Zhong J, Shrier DA, et al. 2001. Diffusion anisotropy of the internal capsule and the corona radiata in association with stroke and tumors as measured by diffusion-weighted MR imaging. AJNR Am J Neuroradiol, 22:456-63.

Hossmann KA, Hoehn-Berlage M. 1995. Diffusion and perfusion MR imaging of cerebral isquemia. Cerebrovasc Brain Metab Rev, 7:187-217.

Huang J, Auchus AP. 2007. Diffusion tensor imaging of normal appearing white matter and its correlation with cognitive functioning in mild cognitive impairment and Alzheimer's disease. Ann NY Acad Sci, 1097:259-64.

Huang J, Friedland RP, Auchus AP. 2007. Diffusion tensor imaging of normal-appearing white matter in mild cognitive impairment and early Alzheimer disease: preliminary evidence of axonal degeneration in the temporal lobe. AJNR Am J Neuroradiol, 28:1943-8.

Jack CR, Petersen RC, O'Brien PC, et al. 1992. MR-based hippocampal volumetry in the diagnosis of Alzheimer's disease. Neurology, 42:183-8.

Jack CR, Petersen RC, Xu YC, et al. 1997. Medial temporal atrophy on MRI in normal aging and very mild Alzheimer's disease. Neurology, 49:786-94.

Kalaria RN. 2000. The role of cerebral ischemia in Alzheimer's disease. Neurobiol Aging, 21:321-30.

Kalaria RN. 2002. Small vessel disease and Alzheimer's dementia: Pathological considerations. Cerebrovasc Dis, 13 (Suppl 2):48-52. 
Kalus P, Slotboom J, Gallinat J, et al. 2006. Examining the gateway to the limbic system with diffusion tensor imaging: the perforant pathway in dementia. Neuroimage, 30:713-20.

Kantarci K, Jack CR, Xu YC, et al. 2001. Mild cognitive impairment and Alzheimer's disease: Regional diffusivity of water. Radiology, 219:101-7.

Killiany RJ, Gomez-Isla T, Moss M, et al. 2000. Use of structural magnetic resonance imaging to predict who will get Alzheimer's disease. Ann Neurol, 47:430-9.

Killiany RJ, Hyman BT, Gomez-Isla T, et al. 2002. MRI measures of entorhinal cortex vs hippocampus in preclinical AD. Neurology, 58:1188-96.

Lansberg MG, Albers GW, Beaulieu C, et al. 2000. Comparison of diffusionweighted MRI and CT in acute stroke. Neurology, 54:1557-61.

Lansberg MG, O'Brien MW, Tong DC, et al. 2001. Evolution of cerebral infarct volume assessed by diffusion-weighted magnetic resonance imaging. Arch Neurol, 58: 613-7.

Le Bihan D, Mangin JF, Poupon et al. 2001. Diffusion tensor imaging: concepts and applications. J Magn Reson Imaging, 13:534-46.

Lutsep HL, Albers GW, DeCrespigny A, et al. 1997. Clinical utility of diffusion-weighted magnetic resonance imaging in the assessment of ischemic stroke. Ann Neurol, 41:574-80.

Medina DA, Stebbins GT, deToledo-Morrell L, et al. 2005. DTI detects dissociated patterns on regional distribution of white matter changes in the elderly. Program No. 542.13. 2005 Abstract Viewer/Itinerary Planner. Washington, DC: Society for Neuroscience [online].

Medina D, deToledo-Morrell L, Urresta F, et al. 2006. White matter changes in mild coginitve impairment and AD: a difusion tensor imaging study. Neurobiol Aging, 27:663-72.

Minoshima S, Giordani B, Berent S, et al. 1997. Metabolic reduction in the posterior cingulate cortex in very early Alzheimer's disease. Ann Neurol, 42:85-94.

Makris N, Worth AJ, Sorenson AG, et al. 1997. Morphometry of in vivo human white matter association pathways with diffusion-weighted magnetic resonance imaging. Ann Neurol, 42:951-62.

Moseley ME, Cohen Y, Mintorovitch J, et al. 1990. Early detection of regional cerebral ischemia in cats: comparison of diffusion- and T2-weighted MRI and spectroscopy. Magn Reson Med, 14: 330-46.

Mukherjee P, Bahn MM, McKinstry RC, et al. 2000. Differences between gray matter and white matter diffusion in stroke: Diffusion-tensor MR imaging in 12 patients. Neuroradiology, 215:211-20.

Naggara O, Oppenheim C, Rieu D, et al. 2006. Diffusion tensor imaging in early Alzheimer's disease. Psychiatry Res, 146:243-9.

Neumann-Haefelin T, Moseley ME, Albers GW. 2000. New magnetic resonance imaging methods for cerebrovasclular disease: emerging clinical applications. Ann Neurol, 47: 559-70.

Pajevic S, Pierpaoli C. 1999. Color schemes to represent the orientation of anisotropic tissues from diffusion tensor data: application to white matter fiber tract mapping in the human brain [erratum in Magn Reson Med, 2000 43(6):921]. Magn Reson Med, 42:526-40.

Petersen RC, Doody R, Kurz A, et al. 2001. Current concepts in mild cognitive impairment. Arch Neurol, 58:1985-92.

Pierpaoli C, Barnett A, Pajevic S, et al. 2001. Water diffusion changes in wallerian degeneration and their dependence on white matter architecture. Neuroimage, 13:1174-85.

Poupon C, Clark CA, Frouin V, et al. 2000. Regularization of diffusionbased direction maps for the tracking of brain white matter fascicles. Neuroimage, 12: 184-95.

Reiman EM, Caselli RJ, Chen K, et al. 2001. Declining brain activity in cognitively normal apolipoprotein $\mathrm{E} \varepsilon 4$ heterozygotes: A foundation for using positron emission tomography to efficiently test treatments to prevent Alzheimer's disease. Proc Natl Acad Sci USA, 98:334-9.

Reiman EM, Caselli RJ, Yun LS, et al. 1996. Preclinical evidence of Alzheimer's disease in persons homozygous for the epsilon 4 allele for apolipoprotein E. $N$ Engl J Med, 334:752-8.
Rose SE, Chen F, Chalk JB, et al. 2000. Loss of connectivity in Alzheimer's disease: an evaluation of white matter tract integrity with color coded MR diffusion tensor imaging. J Neurol Neurosurg Psychiatry, 69:528-30.

Rose SE, Janke AL, Chalk JB. 2008. Gray and white matter changes in Alzheimer's disease: a diffusion tensor imaging study. J Magn Reson Imaging, 27:20-6.

Rose SE, McMahon KL, Janke AL, et al. 2006. MRI diffusion indices and neuropsychological performance in amnestic mild cognitive impairment. J Neurol Neurosurg Psychatry, 77: 1122-8.

Sandson TA, Felician O, Edelman RR, et al. 1999. Diffusion-weighted magnetic resonance imaging in Alzheimer's disease. Dement Geriatr Cogn Disord, 10: 166-71.

Smith SM, Jenkinson M, Johansen-Berg H, et al. 2006. Tract-based spatial statistics: voxelwise analysis of multi-subject diffusion data. Neuroimage, 31:1487-505.

Stieltjes B, Kaufmann WE, van Zijl PC, et al. 2001. Diffusion tensor imaging and axonal tracking in the human brainstem. Neuroimage, 14:723-35.

Sydykova D, Stahl R, Dietrich O, et al. 2007. Fiber connections between the cerebral cortex and the corpus callosum in Alzheimer's disease: a diffusion tensor imaging and voxel-based morphometry study. Cereb Cortex, 17:2276-82.

Takahashi S, Yonezawa H, Takahashi J, et al. 2002. Selective reduction of diffusion anisotropy in white matter of Alzheimer's disease brains measured by 3.0 Tesla magnetic resonance imaging. Neurosci Lett, 332:45-8.

Thompson PM, Hayashi KM, Dutton RA, et al. 2007. Tracking Alzheimer's disease. Ann NY Acad Sci, 1097: 183-214.

Ulug A, Moore DF, Bojko AS, et al. 1999. Clinical use of diffusion-tensor imaging for diseases causing neuronal and axonal damage. AJNR Am J Neuroradiol, 20:1044-8.

Urresta F, Medina DA, Gaviria M. 2003. Diffusion MRI studies in vascular cognitive impairment and dementia. Rev Bras Psichiatr, 25:188-91.

Valla J, Berndt JD, Gonzalez-Lima F. 2001. Energy hypometabolism in posterior cingulate cortex of Alzheimer's patients: superficial laminar cytochrome oxidase associated with disease duration. $J$ Neurosci, 13:4923-30.

Virta A, Barnett A, Pierpaoli C. 1999. Visualizing and characterizing white matter fiber structure and architecture in the human pyramidal tract using diffusion MRI. Mag Reson Imaging, 17:1121-33.

Wang C, Medina D, Stebbins G, et al. 2006. Changes in the microstructural integrity of parahippocampal white-matter tracts in Alzheimer's disease. 2006 Neuroscience Meeting Planner. Atlanta, GA: Society for Neuroscience [online].

Werring DJ, Toosy AT, Clark CA, et al. 2000. Diffusion tensor imaging can detect and quantify corticospinal tract degeneration after stroke. $J$ Neurol Neurosurg Psychiatry, 69:269-72.

$\mathrm{Xu}$ Y, Jack CR, O'Brien PC, et al. 2000. Usefulness of MRI measures of entorhinal cortex versus hippocampus in AD. Neurology, 54:1760-7.

Yasmin H, Nakata Y, Aoki S, et al. 2008. Diffusion abnormalities of the uncinate fasciculus in Alzheimer's disease: diffusion tensor tractspecific analysis using a new method to measure the core of the tract. Neuroradiology, 504:293-9.

Yoneda Y, Tokui K, Hanihara T, et al. 1999. Diffusion-weighted magnetic resonance imaging: detection of isquemic injury 39 minutes after onset in a stroke patient. Ann Neurol, 45:794-7.

Yoshiura T, Mihara F, Ogomori K, et al. 2002. Diffusion tensor in posterior cingulate gyrus: correlation with cognitive decline in Alzheimer's disease. Neuroreport, 13:2299-302.

Zhang Y, Schuff N, Jahng GH, et al. 2007. Diffusion tensor imaging of cingulum fibers in mild cognitive impairment and Alzheimer disease. Neurology, 68:13-19. 